\title{
Elegant Unsupervised Cross-Modal Hashing
}

This paper was downloaded from TechRxiv (https://www.techrxiv.org).

\section{LICENSE}

CC BY 4.0

SUBMISSION DATE / POSTED DATE

$18-11-2021 / 19-11-2021$

CITATION

Cheung, Yiu-ming; Hu, Zhikai (2021): Elegant Unsupervised Cross-Modal Hashing. TechRxiv. Preprint. https://doi.org/10.36227/techrxiv.17036864.v1

$\mathrm{DOI}$

10.36227/techrxiv.17036864.v1 


\title{
Elegant Unsupervised Cross-Modal Hashing
}

\author{
Zhikai HU, Yiu-ming Cheung
}

\begin{abstract}
Unsupervised cross-modal retrieval has received increasing attention recently, because of the extreme difficulty of labeling the explosive multimedia data. The core challenge of it is how to measure the similarities between multi-modal data without label information. In previous works, various distance metrics are selected for measuring the similarities and predicting whether samples belong to the same class. However, these predictions are not always right. Unfortunately, even a few wrong predictions can undermine the final retrieval performance. To address this problem, in this paper, we categorize predictions as solid and soft ones based on their confidence. We further categorize samples as solid and soft ones based on the predictions. We propose that these two kinds of predictions and samples should be treated differently. Besides, we find that the absolute values of similarities can represent not only the similarity but also the confidence of the predictions. Thus, we first design an elegant dot product fusion strategy to obtain effective inter-modal similarities. Subsequently, utilizing these similarities, we propose a generalized and flexible weighted loss function where larger weights are assigned to solid samples to increase the retrieval performance, and smaller weights are assigned to soft samples to decrease the disturbance of wrong predictions. Despite less information is used, empirical studies show that the proposed approach achieves the state-of-the-art retrieval performance.
\end{abstract}

\section{INTRODUCTION}

With the explosion of multimedia data on the Internet, crossmodal retrieval, which means using data of one modality such as image to retrieve data of other modality such as text, has received considerable attention recently. At the same time, the explosion of multimedia data contributes to stress on the data storage and retrieval efficiency. Thus, many hash-based crossmodal retrieval algorithms have been proposed to alleviate these problems. These approaches project original features of different modalities into a common Hamming subspace and use binary vectors to represent them. Comparing with real-valuebased approaches [1], [2], [3], [4], [5], using binary vectors can save massive space to store the data. On the other hand, the distance between instances can be easily calculated by doing the simple $\mathrm{X}$-OR operation, which improves the retrieval efficiency.

Without label information, unsupervised cross-modal hashing approaches usually need to determine the similarities between multi-modal data first, then use the similarities to supervise the learning of hash codes. Firstly, different distance metrics are designed for measuring the similarities between sample pairs and predicting whether they belong to the same class. After obtaining the intra-modal similarities, they should be fused to obtain the overall inter-modal similarities. As shown in Fig1, based on these inter-modal similarities, samples can be predicted into four bins:

- Extremely similar bin: Samples in this bin are extremely close to the anchor.

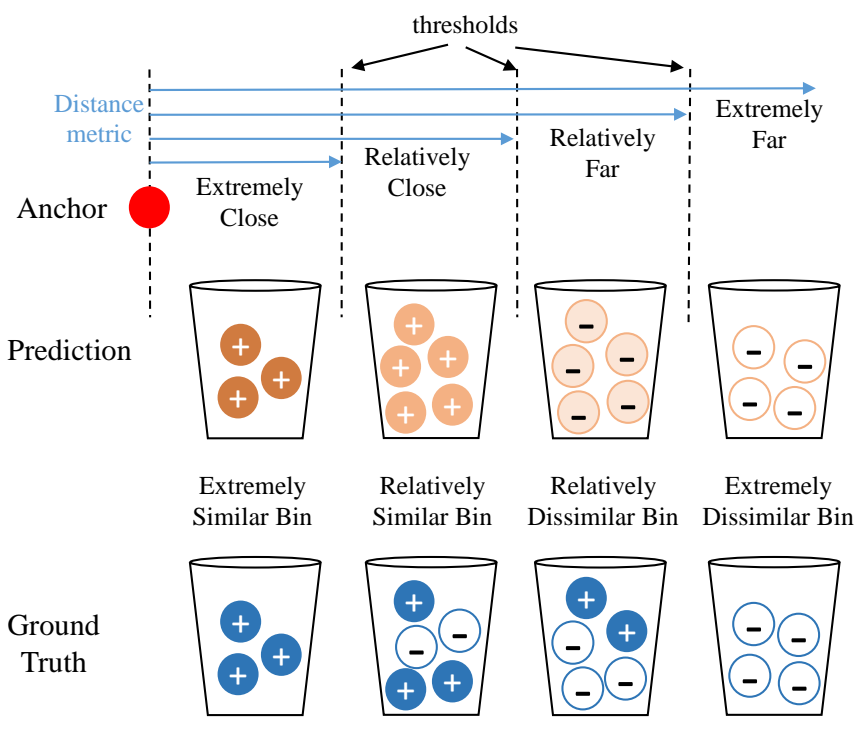

Fig. 1. Predictions with different confidences of samples under certain distance metrix.

- Relatively similar bin: Samples in this bin are relatively close to the anchor.

- Relatively dissimilar bin: Samples in this bin are relatively far from the anchor.

- Extremely dissimilar bin: Samples in this bin are extremely far from the anchor.

Because these predictions are only made by distance, without the help of any label information. Four questions listed below need to be addressed. The first one is the gap between the predictions and the ground truth. Obviously, the gap is determined by the criteria selected for measuring the similarity. If the criteria selected can well unearth the relationships between data, the gap will be small; otherwise, the gap will be huge.

The second one is the definition of relative close and far. Previous works usually utilize the global distribution information to set several thresholds and then determine whether the distances between samples are close or far. However, in practice, we can only use limited data in the training stage. It is therefore extremely hard to get global distribution information.

The third one is the confidence of the prediction in different bins. The gap between predictions and the ground truth could be narrowed but will not disappear. Thus, we need to know whether the prediction is reliable or not. Generally speaking, samples that are extremely close to or far from the anchor are the easiest ones to be right classified. Thus, we can infer that the confidences of predictions of extremely similar and extremely dissimilar are high. We name these predictions as the solid predictions and the samples fall into extremely similar bin and 
extremely dissimilar bin as solid samples. In contrast, samples that are relatively close to or far from the anchor are hard to be right classified, because both positive and negative samples are distributed in this area and usually gather together. Thus, we can infer that the confidences of predictions of relatively similar and relatively dissimilar are low. We name these predictions as the soft predictions and the samples fall into relatively similar bin and relatively dissimilar bin as soft samples.

The last but the most important one is how to utilize solid and soft samples. As mentioned before, the predictions of the solid samples are of high confidence. We can confidently regard them as true-positive or true-negative samples. On the contrary, we should treat soft samples more cautiously because soft predictions are possibly false. One strategy to treat them differently is to artificially increase the similarities of the solid sample to enlarge the gap between solid and soft samples. In other words, this strategy aims to push the extremely similar samples closer to the anchor and pull the extremely dissimilar samples farther from the anchor. Another strategy is to assign larger weights to solid samples and force the model to pay more attention to them.

Many previous works focus on addressing these four problems and achieve satisfactory performance. For example, unsupervised Deep Cross Modal Hashing (UDCMH) [6] adopts the Laplacian constraint to measure the similarities of multimodal data and treats them equally. However, these similarities are not effective enough because they only contain similarity order information. Besides, using this measurement, the global similarities of the whole training set need to be calculated and stored in the memory, which brings high storage and time cost. To tackle these problems, Deep Joint-Semantic Reconstructing Hashing (DJSRH) [7] adopts the absolute distance to measure the similarities between the data within the modalities and fuse them to obtain the inter-modal similarities. This similarity measurement is more effective than the former because it contains more high-order information. However, DJSRH still treats all samples equally, which makes its performance be disturbed by soft samples. Based on DJSRH, Joint-modal Distribution-based Similarity Hashing (JDSH) [8] realizes the differences between solid and soft samples and introduces four kinds of similarities: self-similarity (SS), extreme dissimilarity (ED), relative similarity (RS), and relative dissimilarity (RD). It highlights the importance of samples located in SS and ED area (i.e. solid samples) and proposes a Distribution-based Similarity Decision and Weighting (DSDW) scheme to separate solid and soft samples by artificially increasing similarities of solid samples. However, the DSDW scheme involves massive extra information, including 7 hyper-parameters and global distribution information, and therefore is sensitive to parameters and datasets. Deep Semantic-Alignment Hashing (DSAH) [9] also realizes the differences between solid and soft samples. To emphasize the extremely similar samples, it assigns larger weights to them than other samples, but the partition of weights is fixed, i.e. 2:1. Besides, it ignores the extremely dissimilar samples that are as important as extremely similar samples.

As analyze before, we find that most previous works only focus on addressing part of the four problems we propose. This paper therefore proposes a novel Elegant Unsupervised
Cross-Modal Hashing (EUCMH) method to address these four problems simultaneously. Specifically, we first design a new criterion to measure the similarities of text data, which is more stable in the similarity fusion stage. Then, we find that, under an unsupervised manner, the absolute values of similarities represent not only the similarities but also the confidences of their predictions. We therefore use dot product, instead of the addition with trade-off parameters, to fuse several intra-modal similarities and get the overall inter-modal similarities. Using this fusion strategy, the solid and soft samples can be automatically separated without introducing artificial thresholds. As the absolute values of similarities can represent the confidence of predictions, we directly use them as weights assigned to both solid and soft samples, deriving a more generalized and flexible loss function. In brief, our contributions are listed as follows:

- We define the solid and soft samples and propose to treat them differently. We therefore design a new criterion to measure the similarity of text features and use a simpler fusion strategy to obtain more effective inter-modal similarities. These similarities can not only represent the similarities themselves but also the confidences of the predictions. The definition of close and far can be automatically learned in our fusion strategy. In other words, solid and soft samples can be automatically separated after fusion.

- We propose a more generalized and flexible weighted loss function. which further differentiate solid and soft samples. Besides, our proposed loss function can be transformed into other loss functions easily.

- Although our proposed EUCMH involves fewer hyperparameters and no extra information, empirical studies on two benchmark datasets show that EUCMH achieves the state-of-the-art retrieval performance.

\section{RELATED WORK}

In the literature, most existing hash-based cross-modal retrieval algorithms are supervised ones, e.g., see [10], [11], [12], [13], [14], [15], [16], [17], [18]. SePH [19] learns hash codes by minimizing the KL-divergence between hash codes and the distribution learned from labels. GSePH [20] proposes a more generalized loss function that can handle various crossmodal retreival tasks. SSAH [21] regards label as a new modality and uses it to self-supervise the learning of hash codes. In general, the label information is extremely propitious for improving the discrimination of learned binary representation, but the labels of data are often annotated artificially, whose process is laborious. Further, with the number of multimedia data on the Internet increases dramatically, it becomes very hard or even impossible to annotate all of them. Thus, some researchers shift their sight to unsupervised algorithms.

Without the help of label information, unsupervised hashbased cross-modal retrieval approaches [22], [23] focus more on the properties of multimedia data themselves. They design different criteria to measure the similarities between the data from different modalities, and aim to preserve these similarities in the Hamming subspace. IMH [24] learns hash codes by 


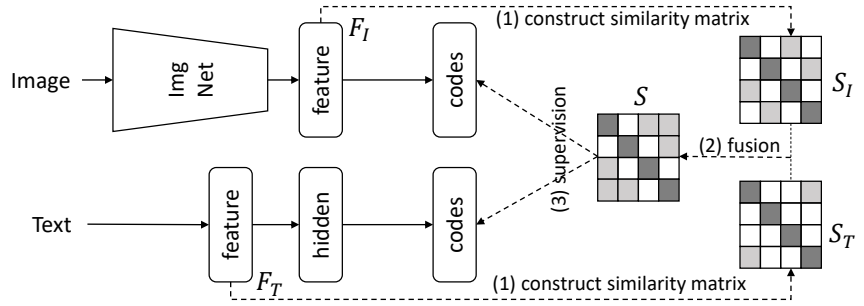

Fig. 2. The overall scheme of proposed EUCMH.

preserving intra- and inter-modal consistency. CMFH [25] uses collective matrix factorization to learn unified hash codes. The multi-modal features these approaches use are hand-crafted, so their performances are not so satisfactory.

As deep neural networks [26], [27], [28], [29] have achieved outstanding performance on feature extraction, many deeplearning-based unsupervised cross-modal approaches have been proposed. Unsupervised Deep Cross Modal Hashing (UDCMH) [6] adopts the Laplacian constraint to preserve the neighborhood similarity of multi-modal data. However, this constraint can only preserve the relative similarity or similarity order. Therefore, it is extremely sensitive to the training samples because different training batches would have different relative relationships. To tackle these problems, Deep Joint-Semantic Reconstructing Hashing (DJSRH) [7] first adopts the absolute distance to measure the similarities between the data within the modalities. Then, it proposes an algorithm to fuse the similarity matrices learned from different modalities and obtains a joint-semantic similarity matrix that contains more high-order information. Based on DJSRH, Joint-modal Distribution-based Similarity Hashing (JDSH) [8] introduces four kinds of multiple similarities to assign different weights to different samples. Furthermore, it investigates the statistical information of the global dataset and proposes a Distribution-based Similarity Decision and Weighting (DSDW) scheme to scale the distances between samples by using the statistical information. Another work inspired by DJSRH is Deep Semantic-Alignment Hashing (DSAH) [9]. It adds two reconstruction layers on the model of DJSRH to reconstruct the original features and a new loss function that contains more constraints to align the semantic information of binary representations of all modalities.

\section{Proposed Method}

\section{A. Problem Definition}

Suppose that there are data of two modalities, their features are denoted as $F_{I} \in \mathbb{R}^{n \times d_{1}}$ and $F_{T} \in \mathbb{R}^{n \times d_{2}}$, where $n$ is the number of data and $d_{1}$ and $d_{2}$ are the dimension of image and text features, respectively. The aim is to learn binary codes $B_{I}, B_{T} \in\{-1,1\}^{n \times k}$ to represent them, respectively, in a common Hamming space, so that they can directly be compared, where $k$ is the hash code length.

The overall scheme of EUCMH is shown in Fig. 2. Firstly, ImgNet is used for extracting image features $F_{I}$, bag-of-words vectors are directly used as the text features $F_{T}$. Secondly, $F_{I}$ and $F_{T}$ are used for calculating the intra-modal similarity matrices $S_{I}$ and $S_{T}$. Thirdly, $S_{I}$ and $S_{T}$ are merged to obtain the overall inter-modal similarity matrix $S$, Finally, $S$ is used for supervising the learning of binary codes $B_{I}$ and $B_{T}$.

\section{B. Construct Similarity Matrices}

In previous works, the cosine distance is usually selected for measuring the intra-modal similarity of both images and texts. That is, $S_{I}$ and $S_{T}$ are calculated by

$$
S_{*}=\cos \left(F_{*}, F_{*}\right)=\left\{\cos \left(F_{*}^{i, \cdot}, F_{*}^{\cdot, j}\right)\right\}_{i, j=1}^{n},
$$

where $* \in\{I, T\}$. Then, to avoid learning some unchangeable hash bits, we use

$$
S_{*}=2 S_{*}-1
$$

to transform $S_{*}$ into $[-1,1]^{n \times n}$.

To build a more effective inter-modal similarity matrix, we randomly select 32 image-text pairs and visualize their intramodal similarities, inter-modal similarities obtained by DJSRH, JDSH, and DSAH, and the ground truth calculated by the label information, as shown in Fig 3 . There are three observations:

1) Fig $3(a)(b)$ are the similarity matrices of image and text calculated by Eq.(1), respectively. It can be observed that except for the diagonal elements, which represent extremely similar samples, most relationships are predicted as dissimilar (soft predictions), especially for the text modality. This is contrary to the ground truth shown in Fig 3 (d). This validates our claim that the predictions of soft samples are usually of low confidence. One possible reason is that text features are high-dimension and always sparse. Cosine distances between them are usually smaller than 0.5. After transformation of Eq.(2), they become minus and are predicted as dissimilar.

2) Fig $3(\mathrm{e})-(\mathrm{g})$ are the inter-modal similarity obtained by DJSRH, DSAH, and JDSH. It can be seen that the similarity information provided by $S_{T}$ has been faded away after fusion. The inter-modal similarity matrix $S$ mainly preserves the information of $S_{I}$. This is because the cosine similarity of text features is usually small as mentioned before. These small numbers vanish after several operations in the fusion procedure.

3) Comparing $S_{I}$ and $S_{T}$ in Fig 3 (a)(b) with the ground truth Fig 3 (d), we find that the text similarity matrix $S_{T}$ reveals the more ground truth relationships. For example, the square similarity relationship in the right-bottom corner in Fig 3(d) is well unearthed by $S_{T}$ in Fig 3 (b), but it is not obvious in Fig 3 (a). Thus, the information loss of $S_{T}$ in the fusion procedure is a great loss of constructing an effective inter-modal similarity.

From these three observations, we know that: (1) cosine similarity is not suited for measuring the similarity of sparse text features; (2) text similarity information is extremely useful for constructing the overall inter-modal similarity matrix; (3) we should treat soft samples cautiously.

To this end, we propose a direct criterion to measure the similarity of text features. Firstly, we compute the similarity matrix $S_{T}$ by

$$
S_{T}=F_{T} * F_{T}^{T} .
$$




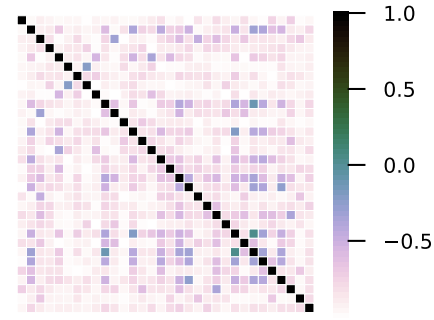

(a) $\mathrm{SI}_{\mathrm{I}}$

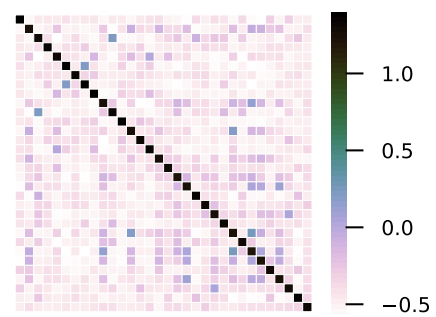

(e) S of DJSRH

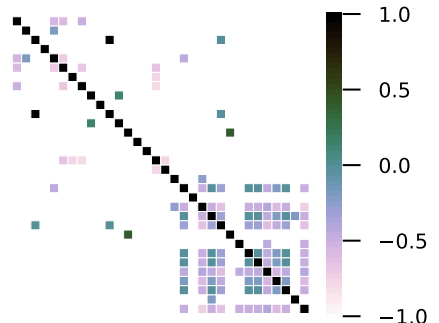

(b) $\mathrm{ST}_{\mathrm{T}}$

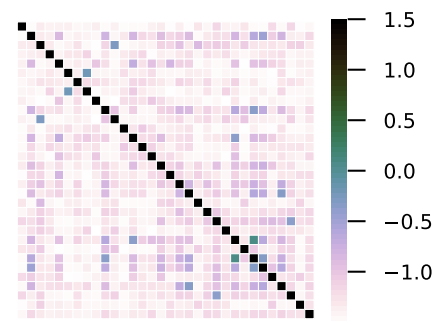

(f) $\mathrm{S}$ of DSAH

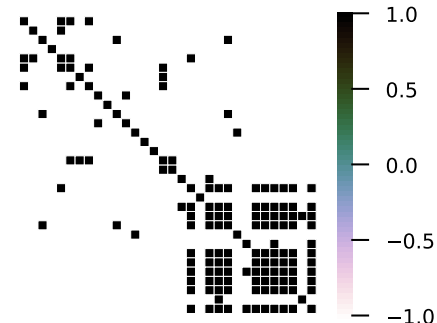

(c) ST of EUCMH

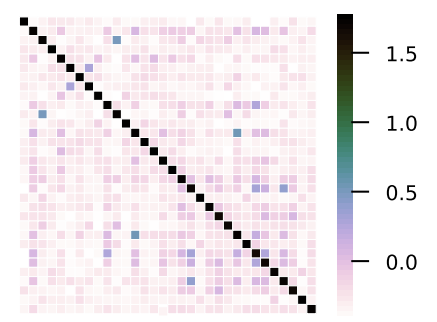

(g) S of JDSH

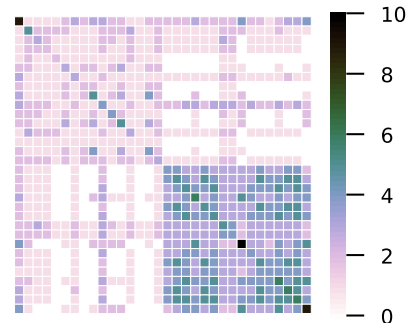

(d) Ground truth

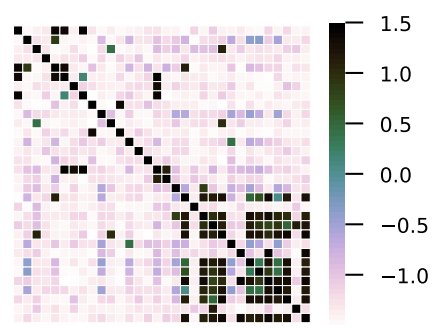

(h) S of EUCMH

Fig. 3. (a) Image similarity matrix $S_{I}$ calculated by Eq.(1). (b) Text similarity matrix $S_{T}$ calculated by Eq.(1). (c) Text similarity matrix $S_{T}$ calculated by Eq.314. (d) Ground truth similarity matrix calculated by labels. (e)-(h) Inter-modal similarity matrix $S$ of DJRSH, DSAH, JDSH, and EUCMH, respectively.

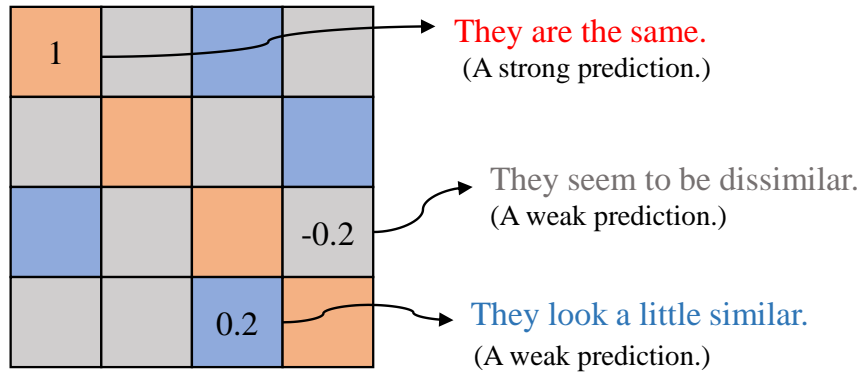

Fig. 4. The absolute values in similarity matrix can be regarded as the confidence of the predictions.

Text descriptions are always presented by short sentences or tag vectors in some wide-used datasets, such as MIR-Flickr [30] and NUS-WIDE [31]. From the practical perspective, if two short sentences or tag vectors share at least one same word or tag, they are probably the descriptions of the same thing. Thus, we refine the $S_{T}$ into $[0,1]$ as:

$$
S_{T}^{i j}= \begin{cases}1, & \text { if } S_{T}^{i j}>0 \\ 0, & \text { otherwise }\end{cases}
$$

After obtaining $S_{I}$ and $S_{T}$, we should fuse them to obtain the overall inter-modal similarity $S$. We find a novel insight that absolute values of elements in similarity matrix represent not only the similarity but also the confidence of their judgements, as shown in Fig 4 . For example, if the value of the similarity is 1 or -1 , it means that corresponding pair is extremely similar or dissimilar with each other. Besides, since its absolute value is 1 , this judgement can be regarded as a strong one. In contrast, if the elements is 0.2 or -0.2 , it means that corresponding pair is not so similar or dissimilar with each other. Meanwhile, since the absolute value of it is 0.2 , this judgement can be regarded as a weak one.
Accordingly, we propose a novel fusion strategy. We use the dot product to obtain the absolute value of $S$ :

$$
S=\left|S_{I} \odot S_{T}\right| .
$$

It is worth noting that, in our fusion strategy, the aim of DSDW scheme can be easily achieved without introducing any statistical information and hyper-parameter. In other words, the solid and soft samples automatically separate during the fusion procedure. Specifically, if the values of both $S_{I}$ and $S_{T}$ are large, which means they are the strong predictions and these samples are solid ones, the values will keep large after dot production, that is, the predictions and samples will keep solid. In contrast, if the values of both $S_{I}$ and $S_{T}$ are small, which means they are the weak predictions and these samples are soft ones, the values will keep small after dot production, that is, the predictions and samples will keep soft. If one of the values of $S_{I}$ and $S_{T}$ are large but another is small, which means one prediction is strong and another is soft, the values will become small after dot production, that is, the solid prediction and sample become soft and the soft ones keep the same. Finally, the gap between solid and soft samples is enlarged.

Then, we need to decide the sign of $S$, because the sign of $S_{I}$ and $S_{T}$ are not always the same, especially for the soft predictions. We adopt an intuitive strategy: if corresponding elements of $S_{I}$ and $S_{T}$ are both positive or negative, i.e., two predictions are accordant, the sign of $S$ will remain the same. However, if one of them is positive but the other is negative, i.e., two predictions are contrary, we should decide the sign of $S$ by the confidences of their predictions. In this way, the sign of $S$ can be calculated by $\operatorname{sign}\left(S_{I}+S_{T}\right)$. Finally, we can obtain the overall inter-modal similarity matrix $S$ by

$$
S=\operatorname{sign}\left(S_{I}+S_{T}\right) \odot\left|S_{I} \odot S_{T}\right| .
$$

By far, the construction of $S$ involves no hyper-parameter and the time complexity of computing it is $O\left(n^{2}\right)$. 


\section{Loss Function}

To further differentiate between solid and soft samples, we decide to assign different weights to them. As we find that the absolute value of similarities can represent the confidences of the predictions, the absolute values of $S$ are the natural weights of each sample. For example, if the absolute value of the element in $S$ is large, it means this prediction is solid, we should assign a large weight to this solid sample. In contrast, if the absolute value of the element in $S$ is small, it means this prediction is soft, we should assign a small weight to this soft sample to avoid the disturbance caused by it. To further highlight the intrinsic connection between image-text pair, finally we obtain the weight matrix as follow:

$$
W=|S|+I,
$$

where $I$ is an identity matrix.

Assigning the weights to different samples, we can obtain a more flexible loss function

$$
\mathcal{L}_{1}=\sum_{* \in\{I, T\}} \sum_{i=1}^{m} \sum_{j=1}^{m} W^{i j}\left(\mu S^{i j}-B_{* *}^{i j}\right)^{2},
$$

where $\mu$ is a parameter to regulate the quantization error.

When $W=1$ (i.e., all-one matrix), it becomes the loss function of DJSRH and JDSH. When $W_{i j}=1, \forall i \neq j$ and $W_{i j}=2, \forall i=j$ (i.e., $W=\mathbf{1}+I$ ), it becomes part of the loss function of DSAH.

To align the semantics of $B_{I T}$, we design another loss function

$$
\mathcal{L}_{2}=\sum_{i=1}^{m} \sum_{j=1}^{m} W^{i j}\left(B_{I T}^{i j}-B_{I T}^{j i}\right)^{2} .
$$

Thus, the overall loss function is $\mathcal{L}=\mathcal{L}_{1}+\mathcal{L}_{2}$.

\section{EXPERIMENT}

\section{A. Datasets}

MIRFlickr [30] consists of 25,000 image-text pairs, i.e. an image and its corresponding textual tags, categorized into 24 concepts. The text of each sample is represented as a 1,386-d bag-of-words vector. For effective training, following [12], we discard the samples whose textual tags are less than 20. Raw images are directly used for training in the model.

NUS-WIDE [31] consists of 260,648 image-text pairs (same as MIRFlickr), categorized into 81 concepts. The text of each sample is represented as a 1,000-d bag-of-words vector. For effective training, following [12], we only select the samples belonging to the 21 most frequent concepts. Raw images are directly used for training in the model.

For both datasets, we follow the setting in [7], [8], [9] and randomly select 2,000 instance as query set. The rest serves as the retrieval set, among which 5,000 instances are randomly selected for training the model.

\section{B. Experimental Set-up}

Baselines. We compare EUCMH with three state-of-the-art unsupervised cross-modal hashing methods DJSRH [7], JDSH [8], and DSAH [9] and several baselines including CVH [22], IMH[24], CMFH [25], LSSH [23] and UDCMH [6].
Evaluation Metric. Two cross-modal retrieval tasks are used for testing: using image to retrieve text $(I \rightarrow T)$ and vice versa $(T \rightarrow I)$. Mean Average Precision (mAP) is used for evaluating the retrieval performance of all approaches.

Implement Details For fair comparison, we follow [7], [8], [9] and use the same base model, which consists of a AlexNet [32] (the backbone of ImgNet), a Multilayer Perceptron (MLP, the backbone of TextNet), and two layers for learning the binary codes. The settings of hyper-parameters in DJSRH, JDSH and DSAH follow the values reported in their papers. There is only one hyper-parameter $\mu$ in our approach. Its function has been tested and demonstrated in [7], so we follow their conclusion and set $\mu=1.5$. Codes of these four methods are run on a PC with an NVIDIA Tesla V100-32G. Additionally, we utilize the SGD [33] optimizer with 0.9 momentum and $5 \times 10^{-4}$ weight decay. The learning rates of both image and text net are 0.001 . We run every codes 10 times and report the average score.

\section{Results and Analysis}

Table I shows the average mAP scores of four approaches on two datasets. It can be observed that:

1) Based on the mAP scores, these approaches can be divided into two groups:(1) DJSRH, JDSH, DSAH, and EUCMH; (2) others. The performance of approaches in the first group significantly surpasses those in the second group. The gaps between them are larger than $10 \%$ in most cases. Among them, EUCMH always achieves the best performance.

2) Comparing the approaches in the first group, the performance of DJSRH is the worst. It may be because that it treats both solid and soft samples equally. Both JDSH and DSAH achieve better performance than DJSRH. That is may because that they realize the differences between solid and soft samples and adopt different strategies to enlarge the gap between them. JDSH enlarges the gap by artificially increasing the similarities of solid samples, while DSAH enlarges the gap by assigning larger weights to them. Our EUCMH adopts these two strategies simultaneously and the weights assigned to similarities are more flexible, so it achieves the best performance.

To further analyse the performance of approaches in the first group, we run their codes 10 times and report the average and standard deviation in Fig 5 . It can be easily observed that EUCMH and DSAH are much more stable than DJSRH and JDSH. As we analyse before, the predictions of soft samples are possibly wrong, which would disturb the performance. Specifically, in the training stage, training data are selected randomly. Every time, different soft samples are randomly selected, so the wrong soft predictions are uncontrollable. If fewer wrong soft predictions are selected in the training stage, the performance will be good. Otherwise, the performance will be spoiled by these soft samples. Both EUCMH and DSAH assign larger weights to solid samples, which weakens the disturbance of soft samples, so they perform stably.

Overall speaking, compared with other approaches, EUCMH uses fewer hyper-parameters, less information, and a simpler 
TABLE I

COMPARISON IN TERM OF MAP SCORES OF TWO RETRIEVAL TASKS ON MIRFLICKR AND NUS-WIDE DATASETS. BEST RESULTS ARE MARKED IN BOLD.

\begin{tabular}{|c|c|c|c|c|c|c|c|c|c|}
\hline \multirow{2}{*}{\multicolumn{2}{|c|}{$\mathrm{mAP}$}} & \multicolumn{4}{|c|}{ MIRFlickr } & \multicolumn{4}{|c|}{ NUS-WIDE } \\
\hline & & 16bits & 32bits & 64bits & 128bits & 16bits & 32bits & 64bits & 128bits \\
\hline \multirow{10}{*}{$I \rightarrow T$} & $\mathrm{CVH}$ & 0.498 & 0.496 & 0.496 & 0.495 & 0.263 & 0.303 & 0.315 & 0.320 \\
\hline & IMH & 0.483 & 0.493 & 0.497 & 0.494 & 0.319 & 0.320 & 0.325 & 0.327 \\
\hline & CMFH & 0.517 & 0.516 & 0.529 & 0.535 & 0.353 & 0.366 & 0.361 & 0.377 \\
\hline & LSSH & 0.500 & 0.502 & 0.508 & 0.512 & 0.395 & 0.398 & 0.401 & 0.409 \\
\hline & DBRC & 0.512 & 0.520 & 0.521 & 0.524 & 0.352 & 0.350 & 0.341 & 0.349 \\
\hline & UDCMH & 0.572 & 0.580 & 0.582 & 0.592 & 0.402 & 0.408 & 0.419 & 0.425 \\
\hline & DJSRH & 0.651 & 0.661 & 0.672 & 0.682 & 0.506 & 0.518 & 0.529 & 0.553 \\
\hline & JDSH & 0.676 & 0.686 & 0.694 & 0.697 & 0.555 & 0.574 & 0.586 & 0.598 \\
\hline & DSAH & 0.679 & 0.694 & 0.696 & 0.702 & 0.566 & 0.586 & 0.595 & 0.603 \\
\hline & EUCMH & 0.692 & 0.700 & 0.709 & 0.714 & 0.580 & 0.593 & 0.603 & 0.611 \\
\hline \multirow{10}{*}{$T \rightarrow I$} & $\mathrm{CVH}$ & 0.491 & 0.485 & 0.479 & 0.489 & 0.290 & 0.301 & 0.311 & 0.312 \\
\hline & IMH & 0.486 & 0.493 & 0.496 & 0.498 & 0.322 & 0.323 & 0.325 & 0.321 \\
\hline & CMFH & 0.556 & 0.560 & 0.572 & 0.580 & 0.370 & 0.381 & 0.396 & 0.405 \\
\hline & LSSH & 0.560 & 0.561 & 0.573 & 0.574 & 0.412 & 0.421 & 0.435 & 0.447 \\
\hline & DBRC & 0.519 & 0.520 & 0.524 & 0.530 & 0.359 & 0.359 & 0.378 & 0.383 \\
\hline & UDCMH & 0.569 & 0.571 & 0.575 & 0.586 & 0.433 & 0.447 & 0.460 & 0.468 \\
\hline & DJSRH & 0.653 & 0.660 & 0.677 & 0.673 & 0.519 & 0.513 & 0.555 & 0.568 \\
\hline & JDSH & 0.675 & 0.685 & 0.698 & 0.701 & 0.570 & 0.580 & 0.599 & 0.613 \\
\hline & DSAH & 0.678 & 0.694 & 0.697 & 0.704 & 0.569 & 0.593 & 0.614 & 0.615 \\
\hline & EUCMH & 0.688 & 0.698 & 0.707 & 0.712 & 0.578 & 0.601 & 0.614 & 0.625 \\
\hline
\end{tabular}

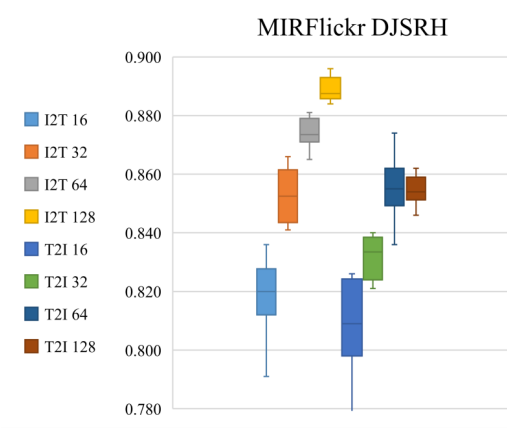

(a)

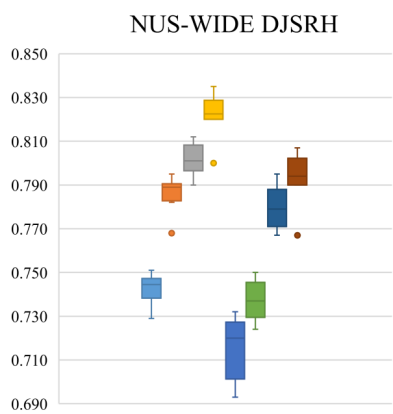

(e)

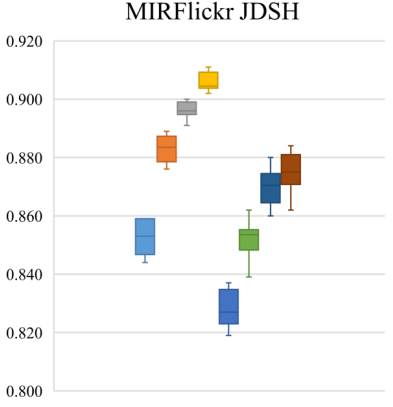

(b)

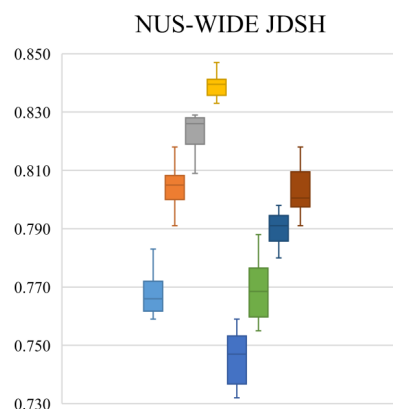

(f)

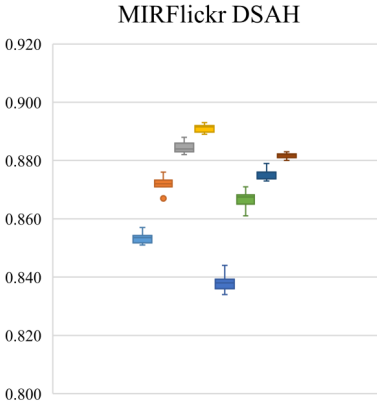

(c)

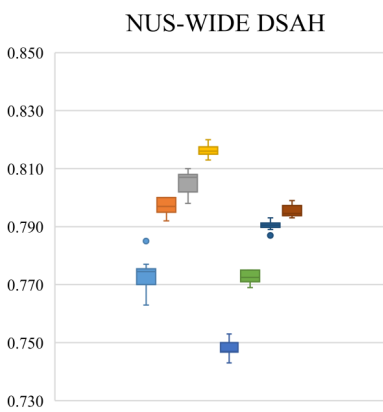

(g)

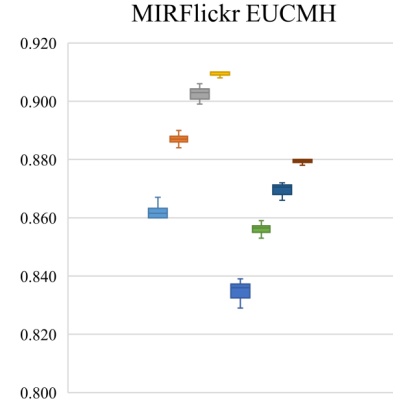

(d)

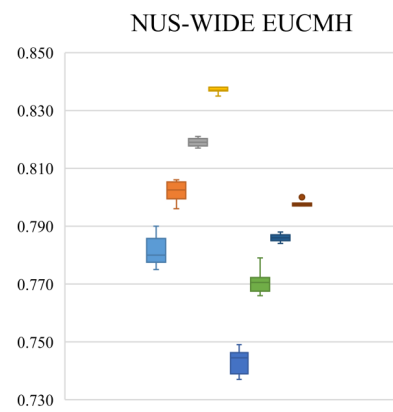

(h)

Fig. 5. The box chart of 10 map@all results of four methods on two datasets.

fusion strategy, but achieves state-of-the-art and stable performance.

\section{Discussion on Similarity Distribution}

To validate the claimant that our proposed fusion strategy can easily achieve the aim of the DSDW scheme in JDSH. We randomly select a training batch and visualize their similarity distributions of JDSH and EUCMH in Fig 6 .

The function of the DSDW scheme is that artificially increase the similarities of solid samples to enlarge the gap between solid and soft samples. It can be observed that both distributions are present in U shape. It means the gap between solid and soft samples indeed be enlarged. However, the similarity distribution of EUCMH is more reasonable:

1) Most similarities are predicted as dissimilar in JDSH, only a few samples are regarded as solid similar samples. Besides, there is a clear blank interval [0.6,1], where soft similar samples should exist. While in EUCMH, the distribution is relatively balanced, a coherent transition of the distribution can be clearly observed.

2) In EUCMH, the values of solid samples are very close 1.5 or -1.5. While in JDSH, most solid dissimilar samples are 


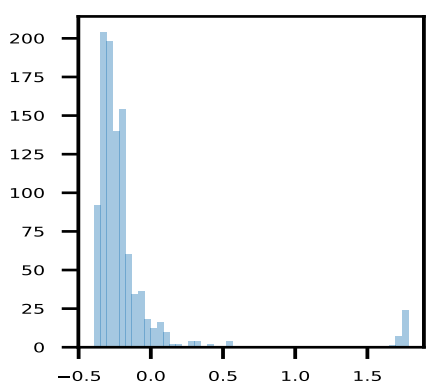

(a) JDSH

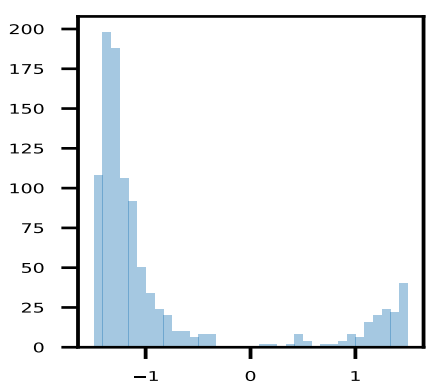

(b) EUCMH
Fig. 6. Distributions of different types of similarity $(\mu=1.5)$.

distributed in $[-0.5,0]$. Generally, samples in this interval can only be regarded as soft samples.

Overall, despite that our proposed fusion strategy involves no hyper-parameter and extra information, it still makes the solid and soft samples separate automatically. Considering that building a DSDW scheme needs 7 hyper-parameters and global distribution information, we can conclude that EUCMH indeed achieves the aim of the DSDW scheme elegantly.

\section{CONCLUSION}

In this paper, we first analyze four key problems in the unsupervised cross-modal retrieval task. To address these problems, we introduce the concepts of the solid and soft predictions and the solid and soft samples. Based on these concepts, we propose to treat solid and soft samples differently. We also find that the values of similarities represent not only the similarities themselves but also the confidences of the predictions. Subsequently, we design an elegant fusion strategy to obtain more effective inter-modal similarities. After fusion, the gap between solid and soft samples is enlarged automatically. Besides, the inter-modal similarities can be directly used as weights to assign different weights to solid and soft samples. Finally, we propose a more generalized and flexible weighted loss function. Experiment results on two datasets indicate that our proposed approach achieves the best retrieval performance and keeps stable.

\section{REFERENCES}

[1] Bokun Wang, Yang Yang, Xing Xu, Alan Hanjalic, and Heng Tao Shen, "Adversarial cross-modal retrieval," in Proceedings of the 25th ACM international conference on Multimedia, 2017, pp. 154-162.

[2] Li He, Xing Xu, Huimin Lu, Yang Yang, Fumin Shen, and Heng Tao Shen, "Unsupervised cross-modal retrieval through adversarial learning," in 2017 IEEE International Conference on Multimedia and Expo (ICME). IEEE, 2017, pp. 1153-1158.

[3] Fangxiang Feng, Xiaojie Wang, Ruifan Li, and Ibrar Ahmad, "Correspondence autoencoders for cross-modal retrieval," 2015.

[4] Liang Zhang, Bingpeng Ma, Guorong Li, Qingming Huang, and Qi Tian, "Multi-networks joint learning for large-scale cross-modal retrieval," in Proceedings of the 25th ACM international conference on Multimedia, 2017, pp. 907-915.

[5] Yunchao Wei, Yao Zhao, Canyi Lu, Shikui Wei, Luoqi Liu, Zhenfeng Zhu, and Shuicheng Yan, "Cross-modal retrieval with cnn visual features: A new baseline," IEEE transactions on cybernetics, vol. 47, no. 2, pp. 449-460, 2016.

[6] Gengshen Wu, Zijia Lin, Jungong Han, Li Liu, Guiguang Ding, Baochang Zhang, and Jialie Shen, "Unsupervised deep hashing via binary latent factor models for large-scale cross-modal retrieval.," in IJCAI, 2018, pp. 2854-2860.
[7] Shupeng Su, Zhisheng Zhong, and Chao Zhang, "Deep joint-semantics reconstructing hashing for large-scale unsupervised cross-modal retrieval," in Proceedings of the IEEE/CVF International Conference on Computer Vision, 2019, pp. 3027-3035.

[8] Song Liu, Shengsheng Qian, Yang Guan, Jiawei Zhan, and Long Ying, "Joint-modal distribution-based similarity hashing for large-scale unsupervised deep cross-modal retrieval," in Proceedings of the 43rd International ACM SIGIR Conference on Research and Development in Information Retrieval, 2020, pp. 1379-1388.

[9] Dejie Yang, Dayan Wu, Wanqian Zhang, Haisu Zhang, Bo Li, and Weiping Wang, "Deep semantic-alignment hashing for unsupervised crossmodal retrieval," in Proceedings of the 2020 International Conference on Multimedia Retrieval, 2020, pp. 44-52.

[10] Zhikai Hu, Xin Liu, Xingzhi Wang, Yiu-ming Cheung, Nannan Wang, and Yewang Chen, "Triplet fusion network hashing for unpaired crossmodal retrieval," in Proceedings of the 2019 on International Conference on Multimedia Retrieval, 2019, pp. 141-149.

[11] Xin Liu, Zhikai Hu, Haibin Ling, and Yiu-ming Cheung, "Mtfh: A matrix tri-factorization hashing framework for efficient cross-modal retrieval," IEEE transactions on pattern analysis and machine intelligence, 2019.

[12] Qing-Yuan Jiang and Wu-Jun Li, "Deep cross-modal hashing," in Proceedings of the IEEE conference on computer vision and pattern recognition, 2017, pp. 3232-3240.

[13] Wen Gu, Xiaoyan Gu, Jingzi Gu, Bo Li, Zhi Xiong, and Weiping Wang, "Adversary guided asymmetric hashing for cross-modal retrieval," in Proceedings of the 2019 on International Conference on Multimedia Retrieval, 2019, pp. 159-167.

[14] Qing-Yuan Jiang and Wu-Jun Li, "Discrete latent factor model for crossmodal hashing," IEEE Transactions on Image Processing, vol. 28, no. 7, pp. 3490-3501, 2019.

[15] Donglin Zhang and Xiao-Jun Wu, "Scalable discrete matrix factorization and semantic autoencoder for cross-media retrieval," IEEE Transactions on Cybernetics, 2020.

[16] Xingzhi Wang, Xin Liu, Zhikai Hu, Nannan Wang, Wentao Fan, and Ji-Xiang Du, "Semi-supervised semantic-preserving hashing for efficient cross-modal retrieval," in 2019 IEEE International Conference on Multimedia and Expo (ICME). IEEE, 2019, pp. 1006-1011.

[17] Zhan Yang, Jun Long, Lei Zhu, and Wenti Huang, "Nonlinear robust discrete hashing for cross-modal retrieval," in Proceedings of the 43rd International ACM SIGIR Conference on Research and Development in Information Retrieval, 2020, pp. 1349-1358.

[18] Xing Xu, Kaiyi Lin, Lianli Gao, Huimin Lu, Heng Tao Shen, and Xuelong $\mathrm{Li}$, "Learning cross-modal common representations by private-shared subspaces separation," IEEE Transactions on Cybernetics, 2020.

[19] Zijia Lin, Guiguang Ding, Mingqing Hu, and Jianmin Wang, "Semanticspreserving hashing for cross-view retrieval," in Proceedings of the IEEE conference on computer vision and pattern recognition, 2015, pp. 3864-3872.

[20] Devraj Mandal, Kunal N Chaudhury, and Soma Biswas, "Generalized semantic preserving hashing for n-label cross-modal retrieval," in Proceedings of the IEEE conference on computer vision and pattern recognition, 2017, pp. 4076-4084.

[21] Chao Li, Cheng Deng, Ning Li, Wei Liu, Xinbo Gao, and Dacheng Tao, "Self-supervised adversarial hashing networks for cross-modal retrieval," in Proceedings of the IEEE conference on computer vision and pattern recognition, 2018, pp. 4242-4251.

[22] Shaishav Kumar and Raghavendra Udupa, "Learning hash functions for cross-view similarity search," in Twenty-second international joint conference on artificial intelligence, 2011.

[23] Botong Wu, Qiang Yang, Wei-Shi Zheng, Yizhou Wang, and Jingdong Wang, "Quantized correlation hashing for fast cross-modal search.," in IJCAI. Citeseer, 2015, pp. 3946-3952.

[24] Jingkuan Song, Yang Yang, Yi Yang, Zi Huang, and Heng Tao Shen, "Inter-media hashing for large-scale retrieval from heterogeneous data sources," in Proceedings of the 2013 ACM SIGMOD International Conference on Management of Data, 2013, pp. 785-796.

[25] Guiguang Ding, Yuchen Guo, and Jile Zhou, "Collective matrix factorization hashing for multimodal data," in Proceedings of the IEEE conference on computer vision and pattern recognition, 2014, pp. 20752082.

[26] Kaiming He, Xiangyu Zhang, Shaoqing Ren, and Jian Sun, "Deep residual learning for image recognition," in Proceedings of the IEEE conference on computer vision and pattern recognition, 2016, pp. 770778.

[27] Gao Huang, Zhuang Liu, Laurens Van Der Maaten, and Kilian Q Weinberger, "Densely connected convolutional networks," in Proceedings 
of the IEEE conference on computer vision and pattern recognition, 2017, pp. 4700-4708.

[28] Christian Szegedy, Sergey Ioffe, Vincent Vanhoucke, and Alexander Alemi, "Inception-v4, inception-resnet and the impact of residual connections on learning," in Proceedings of the AAAI Conference on Artificial Intelligence, 2017, vol. 31.

[29] Christian Szegedy, Vincent Vanhoucke, Sergey Ioffe, Jon Shlens, and Zbigniew Wojna, "Rethinking the inception architecture for computer vision," in Proceedings of the IEEE conference on computer vision and pattern recognition, 2016, pp. 2818-2826.

[30] Mark J Huiskes and Michael S Lew, "The mir flickr retrieval evaluation," in Proceedings of the 1st ACM international conference on Multimedia information retrieval, 2008, pp. 39-43.

[31] Tat-Seng Chua, Jinhui Tang, Richang Hong, Haojie Li, Zhiping Luo, and Yantao Zheng, "Nus-wide: a real-world web image database from national university of singapore," in Proceedings of the ACM international conference on image and video retrieval, 2009, pp. 1-9.

[32] Alex Krizhevsky, Ilya Sutskever, and Geoffrey E Hinton, "Imagenet classification with deep convolutional neural networks," Advances in neural information processing systems, vol. 25, pp. 1097-1105, 2012.

[33] Ilya Sutskever, James Martens, George Dahl, and Geoffrey Hinton, "On the importance of initialization and momentum in deep learning," in International conference on machine learning. PMLR, 2013, pp. 11391147. 